\title{
Electronic Screening-Enhanced Hole Pairing in Two-Leg Spin Ladders Studied by High-Resolution Resonant Inelastic X-Ray Scattering at Cu $M$ Edges
}

\author{
A. Rusydi ${ }^{1,2,3,{ }^{*}}$ A. Goos, ${ }^{1}$ S. Binder, ${ }^{1}$ A. Eich,${ }^{1}$ K. Botril,${ }^{1}$ P. Abbamonte,${ }^{4}$ X. Yu, ${ }^{3}$ M. B. H. Breese,${ }^{2,3}$ \\ H. Eisaki, ${ }^{5}$ Y. Fujimaki, ${ }^{6}$ S. Uchida, ${ }^{6}$ N. Guerassimova, ${ }^{7}$ R. Treusch, ${ }^{7}$ J. Feldhaus, ${ }^{7}$ \\ R. Reininger, ${ }^{8}$ M. V. Klein, ${ }^{4}$ and M. Rübhausen ${ }^{1,2, \uparrow}$ \\ ${ }^{1}$ Institut für Angewandte Physik, Universität Hamburg, Jungiusstraße 11, D-20355 Hamburg, Germany \\ and Center for Free Electron Laser Science (CFEL), Notkestraße 85, D-22607 Hamburg, Germany \\ ${ }^{2}$ NUSSNI-NanoCore, Department of Physics, National University of Singapore, Singapore 117542, Singapore \\ ${ }^{3}$ Singapore Synchrotron Light Source, National University of Singapore, Singapore 117603, Singapore \\ ${ }^{4}$ Physics Department and Frederick Seitz, Materials Research Laboratory, University of Illinois, Urbana, Illinois 61801, USA \\ ${ }^{5}$ Nanoelectronics Research Institute, AIST, 1-1-1 Central 2, Umezono, Tsukuba, Ibaraki 305-8568, Japan \\ ${ }^{6}$ Department of Superconductivity, University of Tokyo, Bunkyo-ku, Tokyo 113, Japan \\ ${ }^{7}$ Photon Science, DESY, Notkestraße 85, D-22607 Hamburg, Germany \\ ${ }^{8}$ Advanced Photon Source, Argonne National Laboratory, Argonne, Illinois 60439, USA
}

(Received 7 January 2014; published 4 August 2014)

\begin{abstract}
We study the electronic screening mechanisms of the effective Coulomb on-site repulsion in hole-doped $\mathrm{Sr}_{14} \mathrm{Cu}_{24} \mathrm{O}_{41}$ compared to undoped $\mathrm{La}_{6} \mathrm{Ca}_{8} \mathrm{Cu}_{24} \mathrm{O}_{41}$ using polarization dependent high-resolution resonant inelastic x-ray scattering at $\mathrm{Cu} M$ edges. By measuring the energy of the effective Coulomb on-site repulsion and the spin excitations, we estimate superexchange and hopping matrix element energies along rungs and legs, respectively. Interestingly, hole doping locally screens the Coulomb on-site repulsion reducing it by as much as $25 \%$. We suggest that the increased ratio of the electronic kinetic to the electronic correlation energy contributes to the local superexchange mediated pairing between holes.
\end{abstract}

DOI: 10.1103/PhysRevLett.113.067001

As pointed out by Hubbard, electronic screening in condensed matter systems is an example for a correlation effect [1]. Recent examples of the importance of electronic screening processes are the phase transitions in correlated materials such as in $\mathrm{C}_{60}$ [2] and in the $\mathrm{BaFe}_{2} \mathrm{As}_{2}$ family [3].

Quasi-one-dimensional spin-ladder compounds (SLCs) such as $(\mathrm{La}, \mathrm{Sr})_{14-x} \mathrm{Ca}_{x} \mathrm{Cu}_{24} \mathrm{O}_{41}$ are an ideal example to study electronic screening effects of correlations energies; i.e., correlation energies are lower when electronic screening of Coulomb interactions is present. The SLCs have as a key-structural element copper-oxygen bonds that form $\mathrm{Cu}_{2} \mathrm{O}_{3}$ ladders and $\mathrm{CuO}_{2}$ chains [4]. They contain the basic physics given by the competition between the kinetic energy determined by the hopping matrix elements $t$, the electron-electron interaction (or electronic correlation) energies driven by on-site Coulomb repulsions, and superexchange energies $J$. Indeed, SLCs show striking properties: superconductivity under high pressure [5], a gapped spin-liquid state [6], charge-density waves [7-11], as well as a paired-hole state $[12,13]$. This rich physics is a result of competing energy scales that foster a large number of nearly degenerate ground states [13-17].

In this Letter, we study in unprecedented detail the screening of electronic correlation energies, superexchange, and kinetic energies in undoped $\mathrm{La}_{6} \mathrm{Ca}_{8} \mathrm{Cu}_{24} \mathrm{O}_{41}$ (LCCO) and hole-doped $\mathrm{Sr}_{14} \mathrm{Cu}_{24} \mathrm{O}_{41}$ (SCO). We have developed and used a vacuum-ultraviolet (VUV) off-axis
PACS numbers: 74.78.-w, 71.27.+a, 71.45.Gm, 74.25.Jb

parabolic double-monochromator Raman spectrometer for high-resolution resonance inelastic x-ray scattering (RIXS) at the Free Electron Laser Hamburg (FLASH) of DESY. This allows us to measure and distinguish magnetic and phonon excitations with orbital selectivity at the $\mathrm{Cu} M_{3}$ edges $(\mathrm{Cu}-3 p \rightarrow 3 d)$ and the $\mathrm{Cu} M_{1}$ edge $(\mathrm{Cu}-3 s \rightarrow 4 p)$, and thus to reveal the electronic correlation and superexchange energies, which are then used to determine the hopping matrix elements in LCCO and SCO. The energy resolution was set to $\sim 24 \mathrm{meV}$. We have used sandblasted $\mathrm{Si}$ as the reference to further define elastic, Stokes, and anti-Stokes lines for calibration purposes. All RIXS spectra were corrected to the FLASH intensity. The samples were characterized using resonant soft $\mathrm{x}$-ray scattering, $\mathrm{x}$-ray absorption [9,11,12], and UV-Raman spectroscopy [13]. More details on the RIXS measurements are shown in the Supplemental Material [18].

Figures 1(a) and 1(b) display x-ray absorption spectroscopy (XAS) and FLASH intensities at the $\mathrm{Cu} M_{3}$ edge and $\mathrm{Cu} M_{1}$ edge from SCO, respectively. Figure 1(c) shows a schematic diagram of the electronic band structure of LCCO and SCO. The bare electronic correlation can be described by the bare Coulomb on-site repulsion $\left(U_{d d} \sim 10-12 \mathrm{eV}\right.$ for cuprates $\left.[2,23,24]\right)$ defined by the $\mathrm{Cu}-3 d$ orbitals. This splits $\mathrm{Cu}-3 d$ states exhibiting the bare lower Hubbard band (LHB) and the bare upper Hubbard band (UHB). However, a downfolding of the Hamiltonian, 


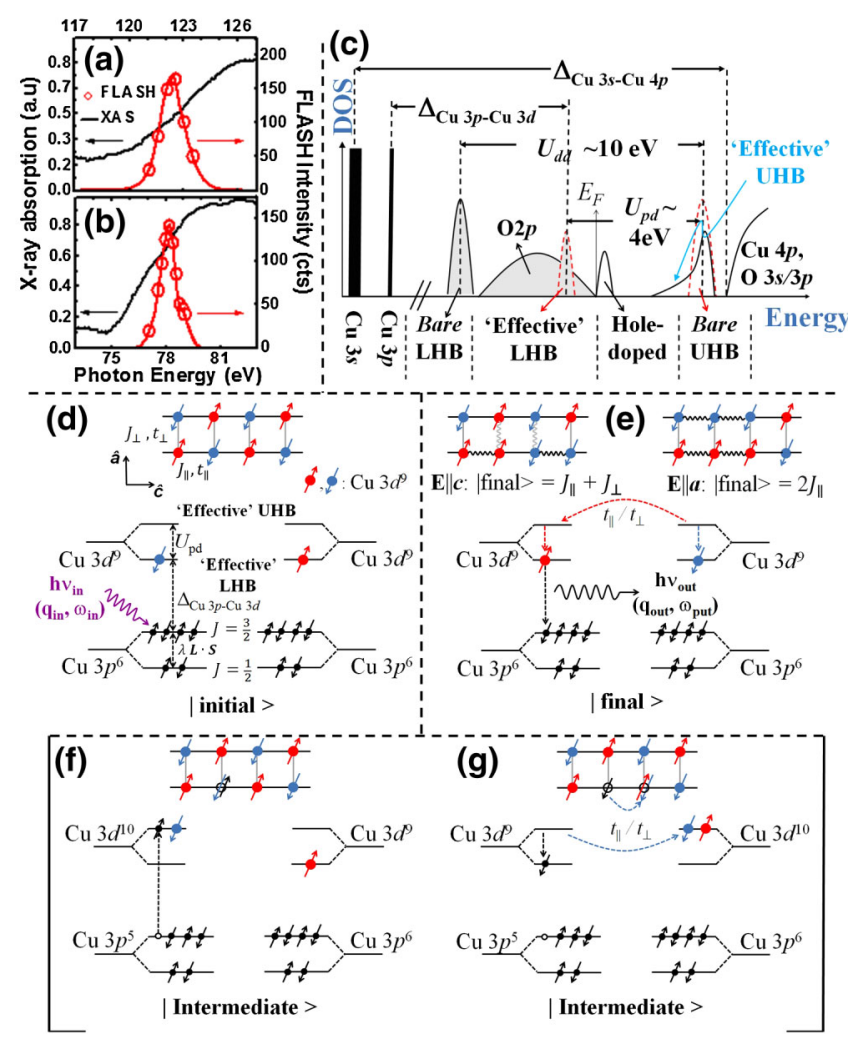

FIG. 1 (color online). X-ray absorption at (a) $\mathrm{Cu}-3 p \rightarrow \mathrm{Cu}-3 d$ and (b) $\mathrm{Cu}-3 s \rightarrow \mathrm{Cu}-4 p \mathrm{M}$-edge transitions and incident photon energies selected for resonance Raman scattering (open bullets) together with the "white-light" intensity distribution of FLASH (red lines). (c) Proposed electronic band structure of the spinladder compounds (SLCs) denoting the relevant energy scales for transitions from the $\mathrm{Cu}-3 p$ and $\mathrm{Cu}-3 s$ states as well as the correlation energies splitting the states close to the Fermi level. Occupied states are shaded in gray. Dashed lines denote the undoped case, with no accessible electronic states at the Fermi level $\left(E_{F}\right)$. (d) The spin ladder with its different superexchange parallel to the leg (along $\hat{c}$ ) and parallel to the rung (along $\hat{a}$ ) of the ladder. (e) The excited final spin state representing the excitation of two magnons. (f) and (g) Intermediate states after absorption of the incident photon outlining the relevant electronic processes between neighboring $\mathrm{Cu}$ sites.

i.e., representing electrons from high energy bands by effective electrons from low energy bands, would screen $U_{d d}$ yielding to a reduced effective Coulomb on-site repulsion [25]. In this scenario, the effective low energy band needs to be clearly separated and decoupled from high energy degrees of freedom and, very importantly, there is only one dominant effective correlation energy, and without involving O- $p$ orbitals. On the other hand, in SLCs the O- $2 p$ bands are closest to the Fermi level $[9,12]$ and thus may strongly affect the effective low energy bands. Strong hybridizations between $\mathrm{Cu}-3 d$ and $\mathrm{O}-2 p$ orbitals $[15,23]$ influence $U_{d d}$ and further modify the bare LHB and bare UHB, normalizing them to an effective LHB and effective $\mathrm{UHB}$, respectively. We call this $U_{p d}$, an effective electronic correlation energy due to strong $\mathrm{Cu}-3 d$ and $\mathrm{O}-2 p$ hybridization. This view is further supported by the fact that in doped SCO the holes have more O- $2 p$ character $[9,12]$. Theoretically, but yet to be proven experimentally, doping should, therefore, result in a significant change in $U_{p d}$ and this can, in principle, yield an anisotropic $U_{p d}$ [25]. Our $M$-edge RIXS measurement aims to reveal $U_{p d}$ and its anisotropy, if any, for undoped LCCO and doped SCO.

By tuning the incoming photon energy to the $M_{3}$ edge, effective electronic correlation energies can be studied through the so-called resonance effect. Hereby, we are accessing spin excitation via matrix elements outlined in Figs. 1(d) $-1(\mathrm{~g})$ as discussed later. Upon doping, the $\mathrm{O}-2 p$ orbitals host the holes $[9,11]$. Furthermore, because the $\mathrm{Cu}-3 d$ orbitals hybridize with the $\mathrm{O}-2 p$ orbitals close to the Fermi level [see Fig. 1(c)], they determine the properties of the low-energy electronic states $[12,26]$. In contrast, the $\mathrm{Cu}-4 p$ orbitals reside well above the Fermi level and hybridize with the $\mathrm{O}-3 p$ orbitals, thus allowing the coupling of photons to oxygen related phonons at the $M_{1}$ edge [see also Fig. 1(c)].

Figure 2(a) highlights the different nature of the excitations for the $\mathrm{Cu} M_{1}$ and $M_{3}$ edge. Indeed, we observe strong phonon excitations for incident photon energies of $\sim 122 \mathrm{eV}$ and spin excitations for incident photon energies of $\sim 80 \mathrm{eV}$. At $\sim 122 \mathrm{eV}$ strong two-phonon scattering can be seen at $120 \mathrm{meV}$ in agreement with visible Raman scattering $[13,27]$. Figure 2(b) shows the high-resolution

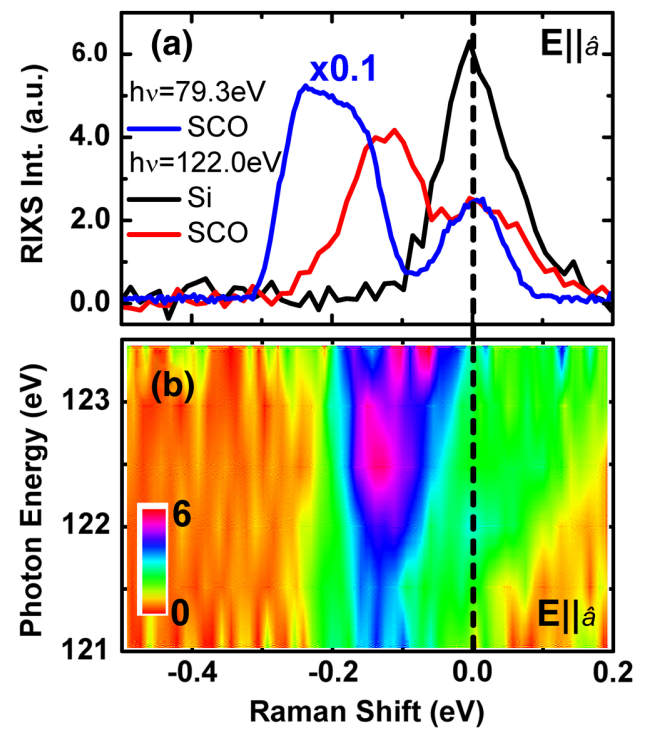

FIG. 2 (color online). (a) Raman spectra (Stokes side is denoted by negative energies) taken at 79.3 and $122.0 \mathrm{eV}$ from the holedoped $\mathrm{Sr}_{14} \mathrm{Cu}_{24} \mathrm{O}_{41}$ (SCO) with the polarization along the rung $(E \| \hat{a})$ and the elastic line from sandblasted $\mathrm{Si}$ for reference. (b) Contour plot of the incident photon energy versus excitation energy (Raman shift) of SCO for $E \| \hat{a}$ showing the resonance behavior of the low-energy phonon excitations. The scale of contour plots is shown. 
resonance profile at the $\mathrm{Cu}-M_{1}$ edge with a resonance energy of $122.5 \mathrm{eV}$. For increasing incident photon energies, we observe the development of one-phonon scattering from oxygen modes below $100 \mathrm{meV}$ Raman shift also in agreement with visible Raman scattering [13,27]. As reference, for $\mathrm{Si}$, we only observe elastic contributions. At $\sim 80 \mathrm{eV}$ higher-energy inelastic scattering can be seen from double spin-flip-two-magnon excitation.

Our main observation relates to the dependence of the spin-flip excitation spectrum on the incident photon energy, polarization, and with doping as shown in Fig. 3. The resonance dependence of the excitation spectrum of LCCO can be seen in Figs. 3(a) and 3(b) as a contour plot for polarizations along the rung $(E \| \hat{a})$ and the leg $(E \| \hat{c})$, respectively. In Figs. 3(c) and 3(d) spectra at selected energies of 78, 79, and $80 \mathrm{eV}$ are displayed for $E \| \hat{a}$ and

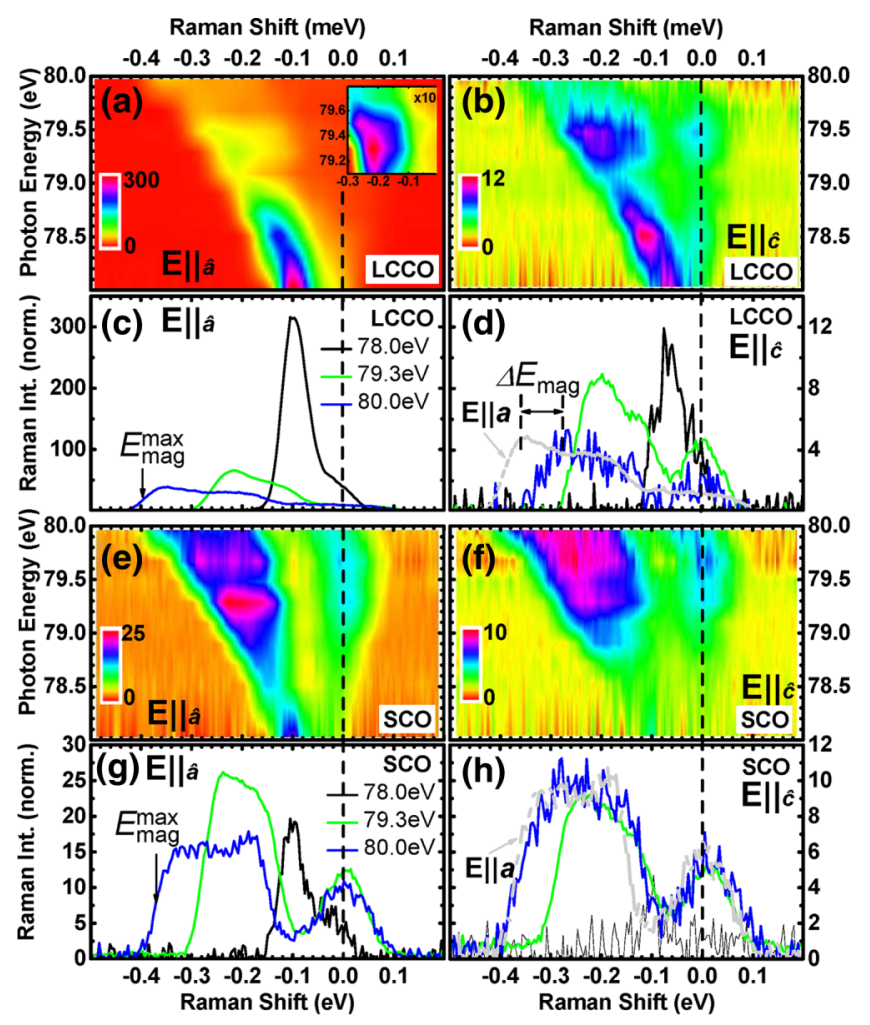

FIG. 3 (color online). Contour plot and resonance profile of resonant inelastic $\mathrm{x}$-ray scattering (RIXS) from undoped $\mathrm{La}_{6} \mathrm{Ca}_{8} \mathrm{Cu}_{24} \mathrm{O}_{41}$ (LCCO) (a),(c) for $E \| \hat{a}$ (along the rungs) and (b),(d) for $E \| \hat{c}$ (along the leg). The inset of (a) enlarges the region of the two-magnon resonance energy. The maximum twomagnon energy is denoted as $E_{\mathrm{mag}}^{\max }$ of about $400 \mathrm{meV}$. The spectrum at $80 \mathrm{eV}$ for $(E \| \hat{c})$ (a gray dotted line) shows the anisotropy of the peak energy denoted as $\Delta E_{\mathrm{mag}}^{\mathrm{max}}$ of about $80 \pm 10 \mathrm{meV}$. Contour plot and resonance profile of resonant inelastic x-ray scattering (RIXS) from doped $\mathrm{Sr}_{14} \mathrm{Cu}_{24} \mathrm{O}_{41}$ (SCO) (e),(g) for $E \| \hat{a}$ polarization and (f),(h) for $E \| \hat{c}$ polarization. In this case the $E_{\mathrm{mag}}^{\max }$ and $\Delta E_{\mathrm{mag}}^{\mathrm{max}}$ are about 375 and $5 \pm 10 \mathrm{meV}$, respectively. For comparison, we replot the E\| $\hat{a}$ spectrum of LCCO and SCO in (d) and (h), respectively.
$E \| \hat{c}$, respectively. For the lowest energy close to the XAS edge at $78 \mathrm{eV}$, a strong excitation is evident at around $90 \mathrm{meV}$ Raman shift that corresponds to a one-magnon excitation [28-30] at the spin-gap energy with momentum transfer $(q) \approx 0$. The one-magnon excitation couples to photons by a finite spin-orbit coupling $(\approx 280 \mathrm{meV})$ of the $\mathrm{Cu}-3 d$ orbitals [31,32]. Interestingly, the one-magnon excitation exhibits a strong anisotropy. For $E \| \hat{a}$ magnetic bonds are broken along the $\hat{c}$ direction, along the leg of the ladder, allowing the propagation of long distance, i.e., small $q$, magnetic excitations [see Figs. 1(d) and 1(e)]. For $E \| \hat{a}$, one-magnon excitations are suppressed due to the geometry of ladders. The one-magnon excitation for LCCO is about 6 times stronger as compared to the two-magnon peak. The inset of Fig. 3(a) displays the sharp resonance of the two-magnon peak $\sim 4 \mathrm{eV}$ above the XAS edge. A strong two-magnon excitation spectrum develops in an energy range up to $E_{\mathrm{mag}}^{\max } \approx 400 \mathrm{meV}$ with a resonance energy of about $79.3 \mathrm{eV}$ as seen in Fig. 3(a) (see inset) and Fig. 3(c).

Based on the Fleury-Loudon Hamiltonian [33] a twomagnon process that considers explicitly a transition of an electron from $\mathrm{Cu}-3 p$ states is shown in Figs. 1(d) and 1(e). In Fig. 1(d) we display the initial state. The $\mathrm{Cu}-3 p$ orbitals are split by the spin-orbit coupling, while the $\mathrm{Cu}-3 d$ orbitals are split by $U_{p d}$. The initial step relies on the dipole allowed transition due to the $(\mathbf{P} \cdot \mathbf{A})$-matrix element from the $\mathrm{Cu}-3 p$ orbital to the unoccupied effective UHB. This results in a doubly occupied site and a hole in the $\mathrm{Cu}-3 p$ state forming a Cu- $3 d^{10}$ and a Cu- $3 p^{5}$ in the intermediate state costing an energy of $\Delta_{\mathrm{Cu} 3 p-\mathrm{Cu} 3 d}+U_{p d}$ being supplied by the incident photon as shown in Figs. 1(f) and 1(g). In the final step we have a transition from effective UHB back into the $\mathrm{Cu}-3 p^{5}$ emitting via the $\mathbf{P} \cdot \mathbf{A}$-matrix element a Raman shifted photon. The energy loss or Raman shift is then given by the effective number of broken spin bonds [cf. Fig. 1(f)]. Within an Ising model each broken bond corresponds to $J / 2$. In two-dimensional antiferromagnetic cuprates this would correspond to $3 \mathrm{~J}$, i.e., the typical energy of a twomagnon excitation. In the SLCs the anisotropy of the kinetic energy needs to be considered. This leads for a hopping along the leg to an energy of $\left(J_{\|}+J_{\perp}\right)$ or for hopping along the rungs to $2 \times J_{\|}$.

The effective scattering Hamiltonian can be projected onto spin states [33] and written as

$$
\begin{aligned}
& H_{\mathrm{int}}(q, \omega)= \frac{\left(t_{\perp / \|}\right)^{2}}{\Delta_{\mathrm{Cu} 3 p-\mathrm{Cu} 3 d}+U_{p d}-\hbar \omega_{\mathrm{inc}}} \\
& \times \sum_{k, q} P(k, q) \vec{S}_{k+q / 2} \cdot \vec{S}_{k-q / 2} \\
& \underset{\substack{\hbar \omega_{\mathrm{inc}} \approx \\
\left(\Delta_{\mathrm{Cu} 3 p-\mathrm{Cu} 3 d}\right)}}{\cong} \sum_{k, q} P(k, q) \vec{S}_{k+q / 2} \cdot \vec{S}_{k-q / 2} .
\end{aligned}
$$

As displayed in Fig. 1(c), $\Delta_{\mathrm{Cu} 3 p-\mathrm{Cu} 3 d}$ is $\sim 75 \mathrm{eV}$. Accordingly, $U_{p d}$ affects the resonance energy of the spin excitation relative to the XAS edge. In the momentum 
transfer $q \rightarrow 0$ limit, we find for incident photon energies close to the energy difference between $\mathrm{Cu}-3 p$ and $\mathrm{Cu}-3 d$ states $\left(\Delta_{\mathrm{Cu} 3 p-\mathrm{Cu} 3 d}+U_{p d} \cong 80 \mathrm{eV}\right)$, the Fleury-Loudon Hamiltonian [33] representing also two-magnon Raman scattering for visible photon energies. Furthermore, Donkov and Chubukov [34] as well as Verney, Gingras, and Devereaux [35] have calculated the spin susceptibility from the Fleury-Loudon Hamiltonian for $q \neq 0$ limit concluding that symmetry selection rules are still valid for $q \rightarrow 0$. The dispersion of the two-magnon excitation does not represent the single-particle dispersion, but rather the $q$ dependence of the magnon-magnon interactions through the vertex correction that results in an effective $q$-dependent vertex.

For LCCO, we find surprisingly large anisotropies in intensity and in the energy of the spin-flip excitations denoted by $\Delta E_{\mathrm{mag}} \approx 80 \pm 10 \mathrm{meV}$ due to the anisotropic superexchange $J_{\|(\perp)} \equiv 2 t_{\|(\perp)} / U_{p d}$ between legs (rungs) (see discussion below). Based on $M$-edge RIXS, $U_{p d}$ is isotropic [see Figs. 2(a) and 2(b)].

Upon the doping of holes into the O- $2 p$ state in $\mathrm{SCO}$ [7,24], which is the isostructural doped counterpart of LCCO [4], we find the following striking behavior as shown in Figs. 2(e)-2(h): First, the zone center $(q=0)$ magnetic excitation is strongly suppressed by a factor of 20 in intensity. Second, the maximum energy of the two-magnon excitation is nearly doping independent $E_{\mathrm{mag}}^{\max } \approx 375 \mathrm{meV}$. The anisotropy of the superexchange energy is suppressed, yielding nearly isotropic energies along the legs and along the rungs with $\Delta E \approx 5 \pm$ $10 \mathrm{meV}$ [see Figs. 2(g) and 2(h)]. More importantly, the resonance seen in the two-magnon channel broadens [compare Figs. 2(a) and 2(e)] and develops a low-energy contribution. Since the resonance energy is given as $\left(\Delta_{\mathrm{Cu} 3 p \text {-Cu3d }}+U_{p d}\right)$ this low-energy contribution signals the enhanced screening of $U_{p d}$, i.e., a reduction of the energy of $U_{p d}$, when holes are doped into the O-2p orbital.

This reduction of $U_{p d}$ is a new observation. Screening of correlation energies is most relevant for the understanding of correlated materials $[3,16,36]$. This also implies that the holes doped into the spin ladder enhance locally $J\left(\sim t^{2} / U_{p d}\right)[2,25]$. We would like to point out that calculations of the spin susceptibility from Eq. (1) lead to excitation energies that are within $10 \%$ equal to the estimates from a simple Ising model in two-dimensional cuprates [37]. From both parameters the anisotropic $t$ can then be calculated $[38,39]$.

For the following discussion, we derive the relevant parameters, yielding $t_{\perp}\left(t_{\|}\right)$from the measured values of $U_{p d, \perp}\left(U_{p d, \|}\right)$ and the corresponding $J_{\perp}\left(J_{\|}\right)$from the twomagnon excitation spectrum. In the ladders of SLCs each spin is surrounded by three nearest neighbors yielding three broken bonds with an energy of $J / 2$ in the $S=1 / 2$ case [cf. Fig. 1(e)]. Considering the anisotropy of the hopping integrals $t_{\|}$and $t_{\perp}$, the superexchange anisotropy yields as spin flip energy, $E_{\text {spinflip }}=2\left(J_{\|} / 2\right)+J_{\perp} / 2$ or as
TABLE I. Superexchange along the legs $\left(J_{\|}\right)$and the rungs $\left(J_{\perp}\right)$, hopping matrix element energies along the legs $\left(t_{\|}\right)$and the rungs $\left(t_{\perp}\right)$, and correlation energies along the legs $\left(U_{\|, p d}\right)$ and the rungs $\left(U_{\perp, p d}\right)$ of the undoped spin-ladder $\mathrm{La}_{6} \mathrm{Ca}_{8} \mathrm{Cu}_{24} \mathrm{O}_{41}$ and the hole-doped spin-ladder $\mathrm{Sr}_{14} \mathrm{Cu}_{24} \mathrm{O}_{41}$ extracted from resonant inelastic x-ray scattering at $\mathrm{Cu} M$ edges.

\begin{tabular}{lrr}
\hline \hline & $\mathrm{La}_{6} \mathrm{Ca}_{8} \mathrm{Cu}_{24} \mathrm{O}_{41}$ & $\mathrm{Sr}_{41} \mathrm{Cu}_{24} \mathrm{O}_{41}$ \\
\hline$J_{\|}$ & $140 \pm 10 \mathrm{meV}$ & $128 \pm 10 \mathrm{meV}$ \\
$J_{\perp}$ & $80 \pm 10 \mathrm{meV}$ & $107 \pm 10 \mathrm{meV}$ \\
$t_{\|}$ & $529 \pm 40 \mathrm{meV}$ & $486 \pm 40 \mathrm{meV}$ \\
$t_{\perp}$ & $400 \pm 40 \mathrm{meV}$ & $400 \pm 40 \mathrm{meV}$ \\
$U_{\|, p d}$ & $4.00 \pm 0.03 \mathrm{eV}$ & $3.70 \pm 0.03 \mathrm{eV}$ \\
$U_{\perp, p d}$ & $4.00 \pm 0.03 \mathrm{eV}$ & $3.00 \pm 0.03 \mathrm{eV}$ \\
\hline \hline
\end{tabular}

maximum two-magnon energy $E_{\mathrm{mag}}^{\max }=2 J_{\|}+J_{\perp}$. This coincides for the isotropic two-dimensional case with $3 J$. The results are summarized in Table I. For LCCO, the superexchange energy is anisotropic with $J_{\|}=140 \pm 10$ and $J_{\perp}=80 \pm 10 \mathrm{meV}$ leading to a maximum two-magnon energy of about $360 \pm 10 \mathrm{meV}=E_{\text {mag }}^{\max }$ (see Fig. 3 and also the Supplemental Material [18]). Whereas the effective correlation energy is isotropic with $U_{\|, p d}=U_{\perp, p d} \sim 4.00 \pm$ $0.03 \mathrm{eV}$ resulting in $t_{\|}=529 \pm 40$ and $t_{\perp}=400 \pm$ $40 \mathrm{meV}$, in good agreement with local-density approximation calculations [40]. In SCO, the magnetic peaks become more isotropic leading to $J_{\|}=128 \pm 10$ and $J_{\perp}=107 \pm 10 \mathrm{meV}$. Noting that inelastic neutron scattering [41] and ${ }^{17} \mathrm{O}$ NMR [6] measurements suggested that $J_{\perp}$ and $J_{\|}$are about $72-80$ and $130-160 \mathrm{meV}$, respectively, yielding a higher superexchange anisotropy as compared to the values measured by $M$-edge RIXS. While the $J_{\|}$is close to the value given by inelastic neutron scattering, the $J_{\perp}$ is slightly larger, reducing the overall anisotropy. Another surprising result is the anisotropy of $U_{p d}$ in SCO (cf. Table I). This anisotropy is essentially embedded in the downfolding procedure that would lead to an effective $U$ [25], but is shown here experimentally to be relevant. Since $U_{p d}$ and $J$ vary as a function of doping, $t_{\|}$and $t_{\perp}$ change to $486 \pm 40$ and $400 \pm 40 \mathrm{meV}$, respectively, also in good agreement with model calculations [40]. Thus, the enhancement of $J_{\perp \text {,SCO }}$ is equivalent with the reduction of $U_{p d, \mathrm{SCO}}$ along the $\hat{a}$ direction of about $1 \mathrm{eV}$. This is indeed confirmed by the changes in $U_{p d}$ [compare Figs. 3(a) and 3(e)]. In undoped LCCO $U_{p d}$ is isotropic, whereas in doped SCO the redistribution of holes in the rungs and legs lead to the anisotropic $U_{p d}[12,13]$. A screening contribution of $U_{p d}$ develops predominantly along the rungs, where the holes reside in the O- $p$ orbitals and along which they pair accordingly. This effect is negligible along the legs [compare Figs. 3(e) with 3(f)]. Thus, we argue that the anisotropic screening of $U_{p d}$ along the rung triggers the formation of the paired hole state. The observed asymmetry of $U_{p d}$ upon hole doping will have strong consequences for other more isotropic cuprate systems as well. 
In conclusion, from the polarization dependent RIXS measurements, we obtain for doped and undoped SLCs the effective Coulomb on-site repulsion energy $U_{p d}$, superexchange energies $J$, and estimate anisotropic hopping matrix elements $t$ along the leg and the rung of the ladder. We find that the local pairing of holes is promoted by a local screening of the correlation energy $U_{p d}$. Upon doping holes into the O- $2 p$ states, local screening of $U_{p d}$ leads to a local enhancement of the superexchange and triggers the formation of the paired hole state along the rung. Our result shows the potency of high-resolution RIXS at the $\mathrm{Cu} M$ edges to determine competing energy scales in correlated electron systems.

We would like to acknowledge technical support from Holger Weigelt, Benjamin Schulz, Pelangi Saichu, Jason Lim, and Wong How Kwong, and discussions with Dirk Manske, Wilfried Wurth, George Sawatzky, Jochen Schneider, and Lance Cooper. We acknowledge financial support by BMBF under 05K10GUD as well as DFG through $\mathrm{Ru} 773 / 4-1$ \& 4-2, and by Singapore National Research Foundation under its Competitive Research Funding (NRF-CRP 8-2011-06 and NRF2008NRFCRP002024), MOE Tier 2 (MOE2010-T2-2-121), and FRC and NUS YIA.

*phyandri@nus.edu.sg †michael.ruebhausen@desy.de

[1] J. Hubbard, Proc. R. Soc. A 276, 238 (1963).

[2] M. B. J. Meinders, J. van den Brink, J. Lorenzana, and G. A. Sawatzky, Phys. Rev. B 52, 2484 (1995).

[3] P. Werner, M. Casula, T. Miyake, F. Aryasetiawan, A. J. Millis, and S. Biermann, Nat. Phys. 8, 331 (2012).

[4] E. M. McCarron III, M. Subramanian, J. Calabrese, and R. Harlow, Mater. Res. Bull. 23, 1355 (1988).

[5] K. Kojima, N. Motoyama, H. Eisaki, and S. Uchida, J. Electron Spectrosc. Relat. Phenom. 117-118, 237 (2001).

[6] T. Imai, K. R. Thurber, K. M. Shen, A. W. Hunt, and F. C. Chou, Phys. Rev. Lett. 81, 220 (1998).

[7] G. Blumberg, P. Littlewood, A. Gozar, B. S. Dennis, N. Motoyama, H. Eisaki, and S. Uchida, Science 297, 584 (2002).

[8] B. Gorshunov, P. Haas, T. Rõom, M. Dressel, T. Vuletić, B. Korin-Hamzić, S. Tomić, J. Akimitsu, and T. Nagata, Phys. Rev. B 66, 060508 (2002).

[9] P. Abbamonte, G. Blumberg, A. Rusydi, A. Gozar, P. G. Evans, T. Siegrist, L. Venema, H. Eisaki, E. D. Isaacs, and G. A. Sawatzky, Nature (London) 431, 1078 (2004).

[10] A. Rusydi, P. Abbamonte, H. Eisaki, Y. Fujimaki, G. Blumberg, S. Uchida, and G. A. Sawatzky, Phys. Rev. Lett. 97, 016403 (2006).

[11] A. Rusydi, P. Abbamonte, H. Eisaki, Y. Fujimaki, S. Smadici, N. Motoyama, S. Uchida, Y.-J. Kim, M. Rübhausen, and G. A. Sawatzky, Phys. Rev. Lett. 100, 036403 (2008).

[12] A. Rusydi, M. Berciu, P. Abbamonte, S. Smadici, H. Eisaki, Y. Fujimaki, S. Uchida, M. Rübhausen, and G. A. Sawatzky, Phys. Rev. B 75, 104510 (2007).
[13] A. Rusydi, W. Ku, B. Schulz, R. Rauer, I. Mahns, D. Qi, X. Gao, A. T. S. Wee, P. Abbamonte, H. Eisaki, Y. Fujimaki, S. Uchida, and M. Rübhausen, Phys. Rev. Lett. 105, 026402 (2010).

[14] E. Dagotto, J. Riera, and D. Scalapino, Phys. Rev. B 45, 5744 (1992).

[15] M. B. J. Meinders, H. Eskes, and G. A. Sawatzky, Phys. Rev. B 48, 3916 (1993).

[16] A. Rusydi, R. Rauer, G. Neuber, M. Bastjan, I. Mahns, S. Müller, P. Saichu, B. Schulz, S. G. Singer, A. I. Lichtenstein, D. Qi, X. Gao, X. Yu, A. T. S. Wee, G. Stryganyuk, K. Dörr, G. A. Sawatzky, S. L. Cooper, and M. Rübhausen, Phys. Rev. B 78, 125110 (2008).

[17] P. Phillips, Rev. Mod. Phys. 82, 1719 (2010).

[18] See Supplemental Material at http://link.aps.org/ supplemental/10.1103/PhysRevLett.113.067001, which includes Refs. [19-22], for high-energy resonant inelastic $\mathrm{X}$-ray scattering.

[19] P. Kuiper, J.-H. Guo, C. Såthe, L.-C. Duda, J. Nordgren, J. J. M. Pothuizen, F. M. F. de Groot, and G. A. Sawatzky, Phys. Rev. Lett. 80, 5204 (1998).

[20] X. Yu, O. Wilhelmi, H. O. Moser, S. V. Vidyaraj, X. Gao, A. T. Wee, T. Nyunt, H. Qian, and H. Zheng, J. Electron Spectrosc. Relat. Phenom. 144-147, 1031 (2005).

[21] B. Schulz, J. Bäckström, D. Budelmann, R. Maeser, M. Rübhausen, M. V. Klein, E. Schoeffel, A. Mihill, and S. Yoon, Rev. Sci. Instrum. 76, 073107 (2005).

[22] N. Motoyama, T. Osafune, T. Kakeshita, H. Eisaki, and S. Uchida, Phys. Rev. B 55, R3386 (1997).

[23] J. Zaanen, G. A. Sawatzky, and J. W. Allen, Phys. Rev. Lett. 55, 418 (1985).

[24] K. Wohlfeld, A. M. Oleś, and G. A. Sawatzky, Phys. Rev. B 81, 214522 (2010).

[25] M. Imada and T. Miyake, J. Phys. Soc. Jpn. 79, 112001 (2010).

[26] N. Nücker, M. Merz, C. A. Kuntscher, S. Gerhold, S. Schuppler, R. Neudert, M. S. Golden, J. Fink, D. Schild, S. Stadler, V. Chakarian, J. Freeland, Y. U. Idzerda, K. Conder, M. Uehara, T. Nagata, J. Goto, J. Akimitsu, N. Motoyama, H. Eisaki, S. Uchida, U. Ammerahl, and A. Revcolevschi, Phys. Rev. B 62, 14384 (2000).

[27] A. Gozar, G. Blumberg, B. S. Dennis, B. S. Shastry, N. Motoyama, H. Eisaki, and S. Uchida, Phys. Rev. Lett. 87, 197202 (2001).

[28] M. G. Cottam and D. J. Lockwood, Phys. Rev. B 31, 641 (1985).

[29] M. L. Tacon, G. Ghiringhelli, J. Chaloupka, M. M. Sala, V. Hinkov, M. W. Haverkort, M. Minola, M. Bakr, K. J. Zhou, S. Blanco-Canosa, C. Monney, Y. T. Song, G. L. Sun, C. T. Lin, G. M. D. Luca, M. Salluzzo, G. Khaliullin, T. Schmitt, L. Braicovich, and B. Keimer, Nat. Phys. 7, 725 (2011).

[30] J. Schlappa, T. Schmitt, F. Vernay, V. N. Strocov, V. Ilakovac, B. Thielemann, H. M. Rønnow, S. Vanishri, A. Piazzalunga, X. Wang, L. Braicovich, G. Ghiringhelli, C. Marin, J. Mesot, B. Delley, and L. Patthey, Phys. Rev. Lett. 103, 047401 (2009).

[31] G. Ghiringhelli, L. H. Tjeng, A. Tanaka, O. Tjernberg, T. Mizokawa, J. L. de Boer, and N. B. Brookes, Phys. Rev. B 66, 075101 (2002). 
[32] M. W. Haverkort, Phys. Rev. Lett. 105, 167404 (2010).

[33] P. A. Fleury and R. Loudon, Phys. Rev. 166, 514 (1968).

[34] A. Donkov and A. V. Chubukov, Phys. Rev. B 75, 024417 (2007).

[35] F. H. Vernay, M. J. P. Gingras, and T. P. Devereaux, Phys. Rev. B 75, 020403 (2007).

[36] P. Werner and A. J. Millis, Phys. Rev. Lett. 104, 146401 (2010).

[37] C. M. Canali and S. M. Girvin, Phys. Rev. B 45, 7127 (1992).
[38] B. S. Shastry and B. I. Shraiman, Int. J. Mod. Phys. B 05, 365 (1991).

[39] M. Rübhausen, C. T. Rieck, N. Dieckmann, K.-O. Subke, A. Bock, and U. Merkt, Phys. Rev. B 56, 14797 (1997).

[40] T. F. A. Müller, V. Anisimov, T. M. Rice, I. Dasgupta, and T. Saha-Dasgupta, Phys. Rev. B 57, R12 655 (1998).

[41] R. S. Eccleston, M. Uehara, J. Akimitsu, H. Eisaki, N. Motoyama, and S.-i. Uchida, Phys. Rev. Lett. 81, 1702 (1998). 